\title{
34. Remarks on the Investigation of the Origin of Visceral Cartilages by Means of Staining.
}

\author{
By Mamoru Ichikawa. \\ Zoological Institute, Kyoto Imperial University. \\ (Comm. by C. IshIkAwA, M.I.A., Mar. 13, 1933.)
}

The visceral cartilages in amphibia are derived from the neural crest. The fact is experimentally demonstrated by Stone ('22, '26)* in Amblystoma punctatum; removal of the neural crest from the upper portion of the neural canal leads to the deficiency of certain cartilages, such as mandibular, hyoid and branchial, and transplantation of it to the side of the body results in the production of some cartilages in a heterotopic position.

In the present experiment the fate of crest cells in Hynobius nebulos is followed to the visceral mesectoderm by means of staining after the indication of Vogt. In an early stage of neurula between stages 16 and 18, red and blue spots were alternately stained along the outer margin of the right neural fold with neutral red and Nile blue sulphate. With the advance of development, the stained crest cells were easily observed through the ectoderm to migrate in bands downwards. Removing the outlying ectoderm, they were more distinctly visible. Some 40 embryos were thus examined. In the following 2 of the most typical cases were selected for description.

In the specimen shown in fig. A, 3 red and 2 blue marks were stained at stage 18 on the right side of the neural fold. No retardation nor inhibition of development was observed by this staining and about 20 hours later (fig. $A_{1}$ ) the mandibular arch began to be formed behind the fore-brain. In such an early stage the mandibular mesectoderm, which descends earlier than others, had hardly migrated downwards to its own arch. However, at the next stage (stage 26) or about 74 hours after marking, each group of mesectoderm elongated itself considerably. As is clearly shown in fig. $A_{2}$, a greater part of the mandibular group is derived from the $b_{1}$ area and the $r_{1}$ area is also more or less taken into this group. The hyoid group originates in $b_{1}$ alone. The branchial groups, 2 in number, consist of the cells of $r_{2}$ and of an anterior portion of $b_{2}$, while $r_{3}$ is not involved in the formation of these groups. In the

* Journ. Exp. Zoöl., vol. 35 ; vol. 44. 
course of the next 25 hours the hind branchial group divides itself into two, both being derived from $r_{2}$ except the postero-dorsal border of the posterior group (see fig. $A_{3}$ ). Therefore, when the latter is actually individualized, the fourth branchial group would consist of red and blue cells.

The second specimen was stained at about stage 17 also in 5 areas, but 3 blue and 2 red against 2 blue and 3 red in the preceding case. The stained embryo is shown in fig. B. Although the distribution of two colors is alternatively different, the migration of the stained crest cells took place in the same way as before, that is to say, the mandibular group was derived from the region indicated by $b_{2}$, the hyoid and the first branchial groups from that of $r_{2}$ and the remaining branchial groups from $b_{3}$. In the present case 2 most anterior areas were not taken into the formation of the visceral mesectoderm (see fig. $B_{2}$ ).

Thus the localization of the visceral mesectoderm and the mode of its migration in the early neurula stages of Hynobius are shown. As to the problem how the mesectoderm changes into cartilages, the transplantation and removal of the neural crest have been effected and a study of these as well as of sections of the stained material has been carried out, the results of which will appear on another occasion in the near future.

\section{Explanation of figures.}

Fig. A-dorsal view of neurula at an hour after marking, black representing the regions stained with Nile blue sulphate and stripes those stained with neutral red. $A_{1}$ - profile of the same embryo at about 20 hours later, none of the migrating mesectoderm groups are yet visible. $A_{2}$-the same specimen after 74 hours from marking; mandibular group is isolated, while others are not; of 2 branchial groups existing, the hind one is dividing into the second, third and the fourth mesectoderm. $\mathrm{A}_{3}$-the same individual 25 hours later, 3 branchial group (the first, second and combined third and fourth) become distinct, all having lost the connection with their origin. B-dorsal view of another neurula at half an hour after marking. $B_{1}$-the same embryo at 20 hours later, its state of development, corresponding nearly to that in fig. $A_{1} . B_{2}-$ the same specimen at about 100 hours after marking, corresponding nearly to that in fig. $A_{3}$. 
No. 3.] Remarks on the Investigation of the Origin of Visceral Cartilages.
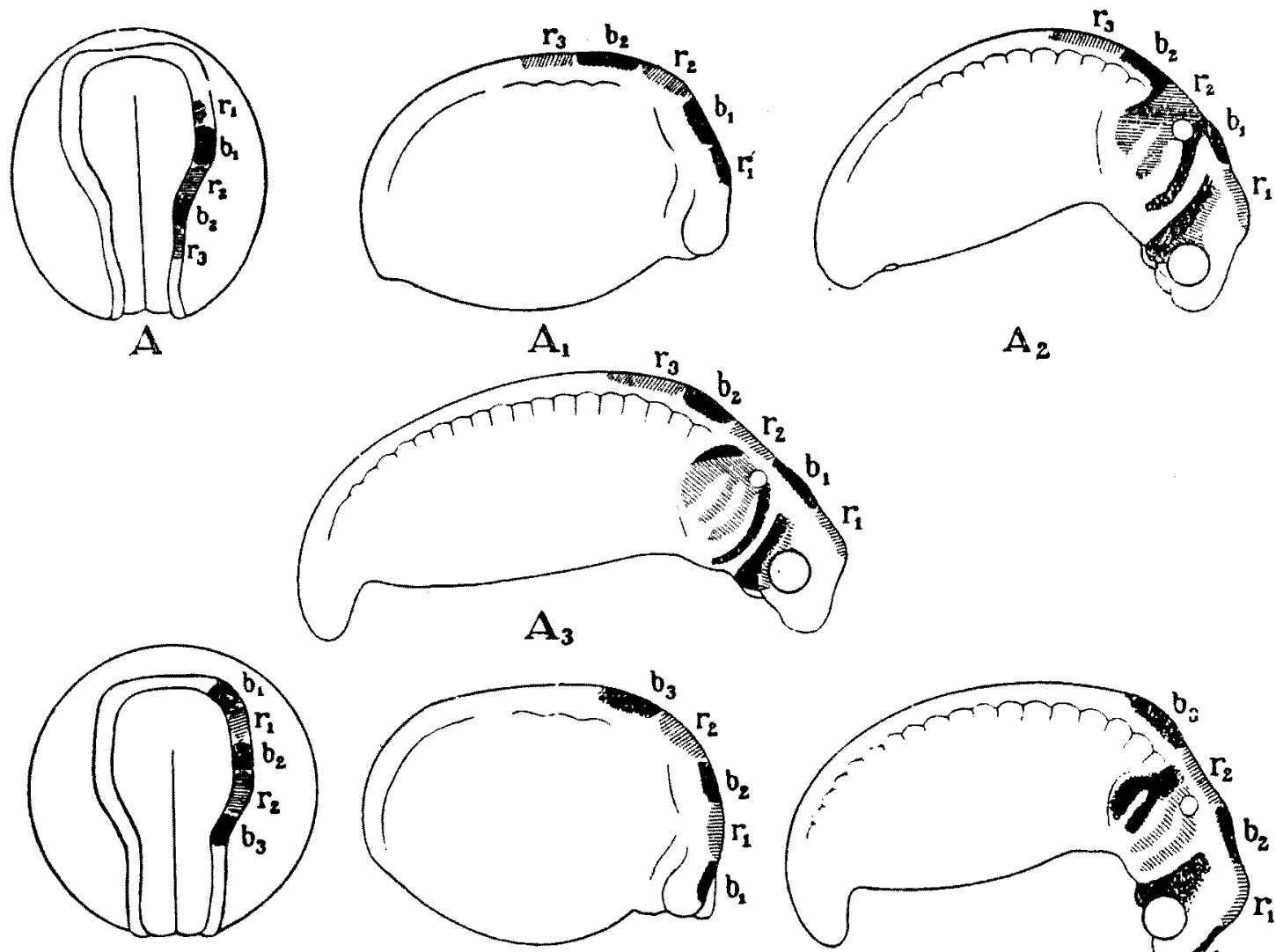

B
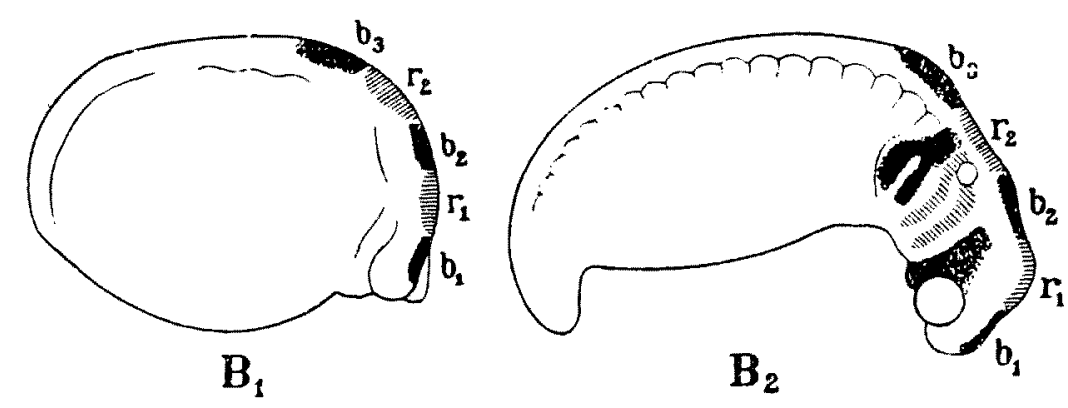